\title{
Monitoring $\beta$-arrestin2 targeting to the centrosome, basal body and primary cilium by fluorescence microscopy
}

Anahi Molla-Herman ${ }^{1,2}$, Kathryn M. Davis ${ }^{3}$, Kirk Mykytyn ${ }^{3,4}$, Alexandre Benmerah $^{5,6}$

${ }^{1}$ Institut Curie, Department of Genetics and Developmental Biology (CNRS-UMR3215, Inserm-U934), 75248 Paris, Cedex 05, France. ${ }^{2}$ CIRB, Collège de France, CNRS-UMR7241, INSERM-U1050, Paris, 75005, France.

${ }^{3}$ Department of Biological Chemistry and Pharmacology, The Ohio State University, Ohio 43210. ${ }^{4}$ Neuroscience Research Institute, The Ohio State University, Ohio 43210.

${ }^{5}$ INSERM U1163, Laboratoire des Maladies Rénales Héréditaires, 75015 Paris, France. ${ }^{6}$ Université Paris Descartes-Sorbonne Paris Cité, Institut Imagine, 75015 Paris, France

Keywords: centrosome, basal body, primary cilia, arrestin, cellular signaling

Running title: Centriolar and ciliary targeting of $\beta$-arrestin2

\section{Corresponding authors:}

Anahi Molla Herman. Email: anahi.molla-herman@ college-de-france.fr

Kirk Mykytyn. Email: Kirk.Mykytyn@osumc.edu

Alexandre Benmerah. Email: alexandre.benmerah@inserm.fr 


\section{Summary}

Primary cilia (PC) are microtubule-based organelles that behave like a cellular antenna controlling key signaling pathways during development and tissue homeostasis. The ciliary membrane is highly enriched for G protein-coupled receptors (GPCRs) and PC are a crucial signaling compartment for this large receptor family. Downstream effectors of GPCR signaling are also present in cilia, and evidence obtained by our labs and others demonstrated that $\beta$-arrestin ( $\beta$ arr) family members are differentially recruited to $\mathrm{PC}$, and have investigated the role of GPCR activation in this process. In this chapter, we provide methods based on fluorescence microscopy on fixed or live cells suitable for investigating targeting and recruitment of $\beta$ arrs at PC. 


\section{INTRODUCTION}

Primary cilia (PC) are antenna-like structures on the surface of most quiescent cell types in vertebrates that control key signaling pathways during development and tissue homeostasis (1). PC dysfunctions linked to mutations in genes encoding for ciliary proteins, have been shown to give rise to a growing number of genetic disorders known as ciliopathies (2).

PC are assembled from the mother centriole of the centrosome (Fig.1), the major microtubule organizing center in interphasic cells, which also plays a critical role in the organization of the mitotic spindle during mitosis (3). The centrosome is composed of two centrioles, the mother, which presents specific appendages, and the daughter. During ciliogenesis, the mother centriole docks onto cellular membranes through its distal appendages and the microtubule doublets from its distal end will extend wrapped into the ciliary membrane to form the axoneme (4). The transition fibers formed by the distal appendages together with the transition zone form a barrier similar to the nuclear pores and limits exchanges between the cytoplasm and ciliary compartment (5). Therefore, all ciliary components (membrane and soluble proteins) must be transported through this barrier by a specific process called IFT (IntraFlagellar Transport). This highly conserved machinery mediates both anterograde and retrograde transport of cargos along the axoneme with the help of both kinesin II and cytoplasmic dynein microtubule-based motors. This bidirectional transport allows the entry of molecules into the ciliary compartment as well as their exit, allowing both recycling of the transport machinery and the release of signaling intermediates to the cell body (6).

A striking observation is that PC are highly enriched for GPCRs that play key roles in their sensory/signaling abilities. This includes Rhodopsin in the outer segment of photoreceptors (a modified PC) and Odor receptors in ciliated olfactory neurons as well as smoothened (smo) during Hh signaling in various cell types and somatostatin receptor subtype 3 (Sstr3) and others in neurons (7,8). Cilia are also highly enriched for downstream signaling components of GPCRs including G proteins and adenylate cyclases (7). The fact that $\beta$ arrs are key regulators of GPCRs at the plasma membrane drove several groups to investigate their potential functions at PC. Indeed, the role of visual arrestin in the regulation of Rhodopsin within the outer segment was previously well established. Early observations pointed to a role of $\beta$ arr2 in the desensitization of odor receptors within cilia on olfactory neurons (9). Ciliary localization of $\beta$ arrs, and especially of $\beta$ arr2, was confirmed by two further studies, which also identified them as partners of $\operatorname{KIF3A}(10,11)$, a subunit of the 
dimeric anterograde IFT motor kinesin II (see above). These studies also indicated potential functions of $\beta$ arrs in the transport of smo to PC (11) as well as in ciliogenesis (10). More recently, a series of interesting studies shed some light on the function and regulation of $\beta$ arr in cilia. First, global analysis of ciliary proteins by proximity labeling revealed that $\beta$ arr2 is exported from cilia through the action of the BBSome (12), a complex working together with IFT to regulate trafficking of cargos, including GPCRs, in and out of cilia $(7,8)$ (Fig.1). Second, $\beta$ arrs (13) and again especially $\beta$ arr2 $(14,15)$ were found to be required for the activation-induced elimination of GPCRs from cilia. Interestingly, in the case of Sstr3, activation by the agonist induces a rapid translocation of $\beta$ arr2 into the cilium (14), further confirming that $\beta$ arr2 likely acts as an adaptor connecting activated GPCRs to the IFT/BBSome machinery for ciliary exit similarly as it does with internalization from the plasma membrane through clathrin-coated pits (16).

Beside these observations linking $\beta$ arrs to ciliary functions, $\beta$ arr2 (10), as well as $\beta$ arr1 (17), were also found at the centrosome in cycling cells, as well as at the basal body of ciliated quiescent cells, in which they colocalize with centriolar markers. Interestingly, $\beta$ arrs were shown to bind to $\alpha$-tubulin and to be required somehow for centriole duplication and/or cytokinesis (17). These data suggest that $\beta$ arrs might be involved in cilia-independent functions at the centrosomes, which remain to be investigated.

Here, we provide different methods for investigating the steady state and dynamic recruitment to centrosome/basal body and cilia in RPE1 and IMCD cells, which are cell lines commonly used in laboratories worldwide to study ciliogenesis, as well as in primary neurons. 


\section{MATERIALS}

\subsection{Cells and cell culture media}

1. RPE1, a human retinal pigment epithelial cell line that stably expresses human telomerase reverse transcriptase (hTERT-RPE1; CLONTECH Laboratories, Inc.; ATCC CRL $4000^{\mathrm{TM}}$ ).

2. HeLa, epithelial cervix carcinoma (ATCC CCL-2'TM).

3. HeLa stably expressing Centrin-GFP (M. Bornens, Institut Curie, Paris, France).

4. mIMCD-3 (IMCD), mouse medulla/collecting duct epithelial cell line (ATCC CRL$\left.2123^{\mathrm{TM}}\right)$.

5. Mouse hippocampal neurons cultured from newborn (P0) pups.

6. Culture media for cell lines: Dulbecco's Modified Eagle's Medium DMEM-F12 1:1 GlutaMAX supplemented with antibiotics (100 U/ml penicillin and $100 \mathrm{mg} / \mathrm{ml}$ streptomycin) and 10\% fetal bovine serum (FBS) for basic RPE1 cell culture conditions or with $0.5 \%$ FBS for low serum containing media. DMEM supplemented with 10\% FBS for HeLa cells. DMEM-F12 1:1 supplemented with 10\% FBS for IMCD cell culture conditions.

7. Ciliation media: culture media with low serum (0.5\% FBS)

8. Neurobasal-A medium (ThermoFisher Scientific) containing B-27 supplement (ThermoFisher Scientific), $0.5 \mathrm{mM}$ GlutaMAX, insulin-transferrin-sodium selenite (ITS, Sigma-Aldrich), $50 \mu \mathrm{g} / \mathrm{ml}$ gentamicin and Hibernate A low fluorescence medium (BrainBits) for cultured neurons.

9. Transfection media for RPE1 cells: Opti-MEM (ThermoFisher Scientific) supplemented with $0.5 \%$ FBS.

\subsection{Buffers, solutions for fluorescence microscopy and drugs:}

1. 1X Phosphate-buffered saline (PBS), $\mathrm{pH} 7.4$.

2. 1X Phosphate-buffered saline with $\mathrm{Ca}^{2+}$ and $\mathrm{Mg}^{2+}(\mathrm{PBS}(\mathrm{Ca} / \mathrm{Mg})), \mathrm{pH}$ 7.4.

3. Fixation solution: $4 \%$ Paraformaldehyde (PFA). 
4. Permeabilization buffer for HeLa and RPE1 cells: $0.1 \%$ Triton X-100, $1 \mathrm{mg} / \mathrm{ml}$ BSA in PBS. Buffer should be passed through a sterile filter $(0.2 \mu \mathrm{m})$ to avoid cellular contamination for long-term storage (see Note 1).

5. Permeabilization buffer for IMCD cells: $0.3 \%$ Triton $\mathrm{X}-100,10 \mathrm{mg} / \mathrm{ml} \mathrm{BSA}, 4 \%$ donkey serum and $0.02 \%$ sodium azide in PBS (see Note 2 ).

6. Blocking buffer for HeLa and RPE1 cells: $1 \mathrm{mg} / \mathrm{ml} \mathrm{BSA} \mathrm{in} \mathrm{PBS}$

7. Blocking buffer for IMCD cells: $10 \mathrm{mg} / \mathrm{ml} \mathrm{BSA}, 4 \%$ donkey serum and $0.02 \%$ sodium azide in PBS.

8. Quenching solution: $50 \mathrm{mM} \mathrm{NH}_{4} \mathrm{Cl}$ in $\mathrm{PBS}$.

9. Mounting media for HeLa and RPE1 cells: PBS-glycerol mix (1:1) using the SlowFade Light Antifade Kit containing DAPI from Molecular Probes (ThermoFisher Scientific).

10. Mounting media for IMCD cells: Immu-Mount (ThermoFisher Scientific).

11. Microtubule depolymerizing solutions: Nocodazole (Sigma). $10 \mu \mathrm{M}$ for immediate depolymerization (1h). $1 \mu \mathrm{M}$ for cell-cycle synchronization (24h).

12. Microtubule stabilizing solution: $10 \mu \mathrm{M}$ Taxol (Paclitaxel).

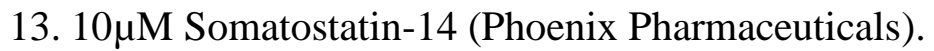

\subsection{Equipment:}

1. Cells are grown in an incubator under standard growth conditions $\left(37^{\circ} \mathrm{C}\right.$ and $\left.5 \% \mathrm{CO}_{2}\right)$.

2. Cells are seeded on $12 \mathrm{~mm}$ glass coverslips (No. 1, NeuVitro).

3. For HeLa and RPE1 cells, coverslips are mounted on plain glass microscope slides (1mm, Pearl). For IMCD cells, coverslips are sandwiched between microscope cover glass (No. 1; ThermoFisher Scientific) and a microscope slide.

4. 6-well or 24-well cell culture plates.

5. 8-well $\mu$-Slide, Ibidi.

6. $\mu$-Dish $35 \mathrm{~mm}$, high Glass Bottom, Ibidi. Neurons were plated on $35 \mathrm{~mm}$ glassbottomed (No. 1.5) dishes (MatTek) that had been coated with $40 \mu \mathrm{g} / \mathrm{ml}$ poly-Dlysine.

7. Electroporation is performed in $0.4 \mathrm{~cm}$ gap Gene Pulser/MicroPulser cuvettes (Bio$\mathrm{Rad})$. 
8. Epifluorescence microscopy: Axiovert 100M (Zeiss) with a micromax camera (Princeton Instruments) driven by Metamorph (Molecular Devices).

9. Confocal microscopy: TCS SP2 AOBS from Leica for FRAP studies in HeLa cells and TCS SP8 from Leica for imaging of IMCD cells.

10. Life-cell confocal microscopy: Andor Revolution WD spinning disk confocal imaging system (Andor Technology plc) controlled by MetaMorph and equipped with a Nikon TiE inverted microscope with Perfect Focus System (Nikon Instruments), Andor iXon Ultra 897 back-illuminated EMCCD camera, ASI XY piezo-Z motorized stage (Applied Scientific Instrumentation), Yokogawa CSU-W1 confocal scanning unit (Yokogawa Electric Corporation) and solid state lasers. OkoLab Bold Line stage-top incubator to control temperature and humidity.

11. Softwares for image analysis: Metamorph (Molecular Devices), ImageJ (https://imagej.nih.gov/ij/), Photoshop (Adobe Systems Inc.), Application Suite X (Leica).

\subsection{Antibodies, staining reagents, plasmids, conjugates and transfection reagents:}

1. Primary antibodies (species, reference number and dilution are indicated):

a. $\beta$ arr2: rabbit pan-arrestin (abcam, ab2914; 1:100) or goat anti-beta arrestin 2 (abcam, ab31294; 1:500).

b. Pericentrin: Rabbit polyclonal (abcam, ab4448), centrosomal marker (1:1000).

c. $\gamma$-tubulin: Mouse monoclonal antibody against $\gamma$-tubulin (clone GTU-88), Sigma, centrosomal marker (1:1000).

d. $\alpha$-tubulin: Mouse monoclonal antibody clone DM1A (T9026), Sigma, microtubule marker $(1: 1000)$.

e. Acetylated- $\alpha$-tubulin (Ac.tub), mouse monoclonal antibody clone 6-11B-1 (Sigma, T7451) marker for stable microtubules $(1: 10,000)$.

f. Flag: rabbit polyclonal antibody from Sigma (F7425) (1:500).

g. Myc: mouse monoclonal antibody clone 9E10 from Santa Cruz Biotechnology (sc40) (1:400).

2. Secondary antibodies:

a. Alexa ${ }^{350}$-conjugated donkey anti-mouse IgG (Invitrogen, A10035) (1:600) 
b. Alexa ${ }^{350}$-conjugated donkey anti-rabbit IgG (Invitrogen, A10039) (1:600)

c. Alexa ${ }^{488}$-conjugated donkey anti-mouse IgG (Invitrogen, A21202) (1:600)

d. Alexa ${ }^{488}$-conjugated donkey anti-rabbit IgG (Invitrogen, A21206) (1:600)

e. Alexa ${ }^{488}$-conjugated donkey anti-goat IgG (Invitrogen, A11055) (1:600)

f. Alexa ${ }^{546}$-conjugated donkey anti-goat IgG (Invitrogen, A10036) (1:1000)

g. Alexa ${ }^{568}$-conjugated donkey anti-goat IgG (Invitrogen, A11057) (1:600)

h. Alexa ${ }^{568}$-conjugated donkey anti-mouse IgG (Invitrogen, A10037) (1:600)

i. Alexa ${ }^{647}$-conjugated donkey anti-mouse IgG (Thermo-fisher, A-31571) (1:600)

j. Alexa ${ }^{647}$-conjugated donkey anti-rabbit IgG (Thermo-fisher, A-31573) (1:600)

3. Expression plasmids (available from our laboratories upon request):

a. $\beta$ arr1-GFP, $\beta$ arr2-GFP and $\beta$ arr2-Cherry were provided by Mark Scott and Stefano Marullo (Institut Cochin, Paris) and previously described in (10).

b. Centrin-GFP was provided by Dr. Michel Bornens (Institut Curie, Paris) (18).

c. Rat wild-type smoothened (smo) cloned into pcDNA3 (Invitrogen) was provided by Dr. J. Coulombe (Institut de Neurobiologie, Gif-sur-Yvette, France). Flag-tagged constitutively active form of smo ( $\left.\mathrm{smo}^{*}\right)$ was generated according to Taipale et al. (19) and described previously in (10).

e. Mouse wild-type somatostatin receptor subtype 3 (Sstr3) was cloned into pcDNA3.1/Myc-His(-) (ThermoFisher) and pDsRed2-N (Takara Bio USA).

4. Transfection reagents: Fugene 6 (Promega), Genejuice (Novagen), Lipofectamine LTX Reagent with PLUS Reagent (ThermoFisher). Cytomix transfection reagent: 120 $\mathrm{mM} \mathrm{KCl}, 0.15 \mathrm{mM} \mathrm{CaCl}_{2}, 10 \mathrm{mM} \mathrm{K} \mathrm{HPO}_{4} / \mathrm{KH}_{2} \mathrm{PO}_{4}, 25 \mathrm{mM}$ Hepes, $2 \mathrm{mM}$ EGTA, 5 $\mathrm{mM} \mathrm{MgCl} 2, \mathrm{pH}$ adjusted to 7.6 with $\mathrm{KOH}$, filter sterilized and stored at $4^{\circ} \mathrm{C}$.

5. Nuclear staining: 4', 6-diamidino-2-phenylindole dihydrochlorid (DAPI) or DRAQ5.

6. Nail polish (electron microscopy science). 


\section{METHODS}

\subsection{Cell cycle synchronization in HeLa cells:}

1. Set down 4 sterilized coverslips per well in a regular 6 well plate under the hood. Add PBS and aspirate to make a vacuum between the coverslips and the plate (see Note 3 ).

2. $1.5 \times 10^{5} \mathrm{HeLa}$ cells are seeded per well in $2 \mathrm{~mL}$ of culture media (section 2.1.6) and cultured for $24 \mathrm{~h}$ (see Note 4 ).

3. In order to increase the number of mitosis to be analyzed per plate, HeLa cells are synchronized with $1 \mu \mathrm{M}$ Nocodazole treatment overnight (see Note 5).

4. Cell cycle arrest is further released by transfer into basic culture media.

5. Wash the cells twice with PBS (see Note 6).

6. Aspirate the PBS and add fresh culture medium ( $2 \mathrm{~mL}$ per well in a 6-well-plate).

7. Cells are analyzed after $6 \mathrm{~h}$ or $12 \mathrm{~h}$ to observe mitotic events.

An example of endogenous $\beta$ arr2 distribution in HeLa cells in interphase and mitosis (metaphase, anaphase and telophase) is shown in Figure 2. Barr2 is localized at the centrosome as shown by its colocalization with the centrosomal marker $\gamma$-tubulin (Fig.2, A and C). $\beta$ arr2 sublocalization at the proximal ends of centrioles was confirmed using Centrin as a marker of the distal ends (Fig.2B).

\subsection{Transfection of HeLa cells with plasmid DNA}

1. Put 4 sterile (autoclaved) coverslips per well in a 6-well plate. Add some PBS and aspirate to make a vacuum between the coverslips and the plate in order to make the coverslips stick to the bottom to avoid coverslip overlapping. Seed $2 \times 10^{5}$ cells per well for $24 \mathrm{~h}$ before transfection (see Note 4 ). Change the culture medium $2 \mathrm{~h}$ before transfection to have an optimal $\mathrm{pH}$ (see Note 6).

2. Prepare the transfection mix: For $1 \mu \mathrm{g}$ of DNA, mix $100 \mu \mathrm{L}$ of serum free culture medium with $3 \mu 1$ of GeneJuice. Vortex and let incubate for 5min at RT. Add $1 \mu \mathrm{g}$ of DNA. Incubate 10-15min at RT for the liposome-DNA complexes to form.

3. Wash the cells twice with PBS (see Note 6).

4. Aspirate the PBS and add fresh culture medium ( $2 \mathrm{~mL}$ per well in a 6-well-plate).

5. Add $100 \mu 1$ of transfection mix per well (see Note 4). 
6. Incubate the cells with the transfection mix for 24 hours before fixation/immunofluorescence or live-cell imaging analysis.

An example of the differential distribution of transfected $\beta$ arrestin GFP fusions in HeLa cells is shown in Figure 3. $\beta$ arr2-GFP is localized at the centrosome as shown by its colocalization with the centrosomal marker pericentrin whereas $\beta$ arr1-GFP shows a cytoplasmic diffuse distribution similar to GFP (Fig.3A).

\subsection{Preparation of cells for immunofluorescence microscopy in RPE1 and HeLa cells}

1. Wash the cells twice with room temperature (RT) PBS (see Note 6)

2. Fix the cells in $4 \%$ PFA for 20 minutes at $4^{\circ} \mathrm{C}$ (by placing the plate on ice), followed by a 10 minutes incubation in quenching solution at RT.

3. Gently wash the cells twice with PBS always avoiding cell detachment (see Note 6)

4. Incubate the cells with primary antibodies in permeabilization buffer for 30-45 minutes at RT. Incubation can be made directly in the well, the minimal mix volume should then be $200 \mu \mathrm{L}$ per well to avoid cells drying. To save antibodies, you can prepare a $40 \mu \mathrm{L}$ drop containing the antibody/permeabilization mix on Parafilm ${ }^{\circledR}$ secured to the bench, and then gently invert your coverslip to put the cells in contact with the liquid. Do not forget to invert the coverslip again when you put it back in the well (see Note 7).

5. Wash the cells twice with blocking buffer at RT and avoid cell detachment (see Note $6)$.

6. Incubate with secondary antibodies (mixed in blocking buffer) for 30 minutes at RT in the dark to preserve the fluorescence.

7. Wash once with blocking buffer and twice with PBS (see Note 6).

8. Lay down your cells on microscope slides in mounting medium $(5 \mu \mathrm{L})$.

9. Add nail polish by putting small drops around the coverslip and then complete the circle once the drops are dry (see Note 8).

10. Store at $4{ }^{\circ} \mathrm{C}$ and keep in the dark until observation.

\subsection{Epifluorescence microscopy in fixed HeLa and RPE1 cells}


Pictures were acquired using a 63x objective (plan-apo; Zeiss) with a micromax camera (Princeton Instruments) driven by Metamorph. The final images are generated using ImageJ (https://imagej.nih.gov/ij/) and Photoshop (Adobe Systems Inc.).

\subsection{Quantification of $\beta$ arrestins centrosomal targeting in HeLa Cells: role of microtubules}

1. Transfected HeLa cells are treated or not with Nocodazole or Taxol $(10 \mu \mathrm{M}, 1 \mathrm{~h})$.

2. Cells are subsequently fixed and stained as described in section 3.3 with antibody against a centrosomal marker (pericentrin).

3. GFP signal at the centrosome is quantified from pictures using Metamorph (or ImageJ).

4. Pericentrin staining is used to define a region corresponding to the centrosome. The option "Auto Threshold for light objects" allows to transform the pericentrin staining into objects.

5. The pericentrin objects are then circled by selecting the option "Create regions around objects"'.

6. The resulting regions are then transferred to the GFP corresponding images, and the fluorescence intensity corresponding to GFP at the centrosome (CE) can be measured.

7. A region of the same size is selected outside the cell to measure the noise of the camera $(\mathrm{CN})$ and another one in the cytoplasm $(\mathrm{CY})$ of the same cell.

8. Normalized fluorescence at the centrosome was then calculated as follows: $\mathrm{NF}=(\mathrm{CE}-$ $\mathrm{CN}) /(\mathrm{CY}-\mathrm{CN})$.

An example of quantitative analyses of GFP, $\beta$ arr1-GFP and $\beta$ arr2-GFP localization at the centrosome in HeLa cells is shown in Figure 3. These analyses show that $\beta$ arr2-GFP is enriched at the centrosome compared to $\beta$ arr1-GFP or GFP (Fig.3, A and D). Interestingly, the enrichment of $\beta$ arr2-GFP at the centrosome is not affected by Nocodazole or Taxol (Fig.3, $\mathrm{C}$ and D) showing that it is independent of microtubules.

\section{6. $\beta$ arr 2 dynamics at the centrosome in HeLa cells: Fluorescence after photo-bleaching (FRAP)}

1. $1 \times 10^{5} \mathrm{HeLa}$ cells are seeded in Ibidi 8 -well $\mu$-slides. 
2. After $24 \mathrm{~h}$, cells are transfected with $\beta$ arr2-GFP expressing plasmids as indicated in 3.2 (see Note 9).

3. The day after, ßarr2 dynamics at the centrosome are analyzed using FRAP (fluorescence recovery after photobleaching). Cells are treated or not with $10 \mu \mathrm{M}$ nocodazole for $1 \mathrm{~h}$ at $37^{\circ} \mathrm{C}$, and analyzed using a confocal microscope after excitation with a 488-nm laser line from an argon laser.

4. A centrosomal region (50x50 pixels) is exposed to two consecutive pulses of 1 second with $100 \%$ of laser intensity, and fluorescence recovery is analyzed for 90 seconds taking an image every 1.3 seconds. A cytoplasmic non-centrosomal region of the same size is also taken into account for quantification and normalizations are performed as described in section 3.4 (see Note 10).

5. Images are analyzed using Metamorph to quantify normalized fluorescence at the centrosome similarly as described in section 3.5 except that the region corresponding to the centrosome is directly circled using Metamorph.

An example of analysis of the dynamics of $\beta$ arr2-GFP at the centrosome by FRAP is shown in Fig.3B. After photobleaching, $\beta$ arr2-GFP signal at the centrosome is rapidly and almost fully recovered indicating that the localization of $\beta$ arr2 at the centrosome results from a dynamic exchange between a centrosomal and a cytoplasmic pool.

\subsection{Induction of ciliogenesis in RPE1 cells}

1. Set down one sterile coverslip per well in a regular 24 well plate under the hood.

2. To optimize cell culture and transfection steps, fix your coverslips to the bottom of the wells by filling each well with $0.5 \mathrm{~mL}$ of PBS and then aspirate it with a $200 \mu \mathrm{L}$ pipet tip to create a vacuum between the coverslip and the plate.

3. Seed $2.5 \times 10^{5}$ cells per well and grow them in RPE1 culture conditions for 24 hours (see Note 4).

4. Wash the cells twice in PBS (see Note 6).

5. Add ciliation media $(0.5 \% \mathrm{FBS})$ for $24-48 \mathrm{~h}$ to allow primary cilia formation.

6. Monitor the efficiency of primary cilia formation by staining with acetylated $\alpha$-tubulin antibody (you should obtain $\sim 80 \%$ of ciliated cells) (see Note 11 ).

\subsection{Transfection of cells with plasmid DNA in RPE1 cells}


1. Seed $1 \times 10^{5}$ cells per well in a 24 well plate containing a coverslip per well, and grow them for 24 hours as indicated in 3.7.

2. Prepare the transfection mix. For 4 wells, mix $1 \mu$ g of DNA with $100 \mu \mathrm{L}$ of Opti-MEM and $3 \mu \mathrm{L}$ of Fugene 6.

3. Wash the cells twice with PBS $\left(\mathrm{Ca}^{2+} / \mathrm{Mg}^{2+}\right)$ avoiding cell detachment (see Note 6).

4. Aspirate the PBS and add $500 \mu \mathrm{L}$ of low serum containing Opti-MEM (0.5\% FBS) to each well (see Note 6)

5. Add $25 \mu 1$ of transfection mix per well (see Note 4).

6. Incubate the cells with transfection mix for 24 hours before fixation/immunofluorescence or live-cell imaging analysis.

An example of RPE1 cell expressing endogenous $\beta$ arr2 at the primary cilia is shown in Fig.4A. Primary cilia are enriched with GPCRs (G protein-coupled receptors) as shown by the localization of a constitutively active form of Smoothened (Smo*) at PC in Fig.4B. Interestingly, exogenous $\beta$ arr2-GFP is not present at the axoneme in the absence of $\operatorname{Smo}^{*}(\varnothing)$, but is highly enriched at the basal body. In the presence of Smo*, $\beta$ arr2-GFP signal is more present at the axoneme (Fig.4C, left panels). To further confirm these results, we used another fluorescence tag (Cherry), observing that $\beta$ arr2-Cherry is localized at the axoneme and the basal body in the absence of Smo*, and is more enriched at the axoneme in the presence of Smo* (Fig.4C, right panels).

\subsection{Transfection of IMCD cells with plasmid DNA}

1. Prepare the electroporation mix and place it on ice. For each transfection, combine a total of $10 \mu \mathrm{g}$ of DNA ( $5 \mu \mathrm{g}$ of $\beta \operatorname{arr} 2 \mathrm{GFP}+5 \mu \mathrm{g}$ of Sstr3Myc) with $400 \mu \mathrm{L}$ cytomix containing freshly added $2 \mathrm{mM}$ ATP and $5 \mathrm{mM}$ glutathione.

2. Resuspend $5 \times 10^{6} \mathrm{IMCD}$ cells in $400 \mu \mathrm{L}$ cytomix, pipet into a cuvette on ice and electroporate using the following settings: $320 \mathrm{~V}, 950 \mu \mathrm{F}, \infty \Omega, 4 \mathrm{~mm}$.

3. Resuspend the cells in fresh IMCD medium and plate onto a $60 \mathrm{~mm}$ dish with coverslips placed on the bottom.

4. Wash and refeed the cells 16-24 hours after transfection.

\subsection{Sstr3-mediated ciliary recruitment of $\beta$ arr2-GFP in IMCD cells}

1. After 40-48 hours, set down one coverslip per well in a regular 24 well plate containing $1 \mathrm{~mL}$ of fresh IMCD medium. Let the cells recover for approximately 1 hour in a tissue culture incubator. 
2. Allow the glass vial containing lyophilized Somatostatin-14 (SST) to warm up to room temperature for at least 20 minutes before resuspending it in water to a final concentration of $1 \mathrm{mM}$. Reconstituted SST should be stored in the glass vial at $4^{\circ} \mathrm{C}$ and is effective for at least one month.

3. Add $10 \mu \mathrm{L}$ of water (vehicle) or SST to each well, for a final concentration of $10 \mu \mathrm{M}$ SST, and return the plate to the incubator for 20 minutes.

\subsection{Preparation of IMCD cells for immunofluorescence microscopy}

1. Aspirate the media and fix the cells in $4 \%$ PFA for 15 minutes at RT.

2. Permeabilize the cells in permeabilization buffer for 10 minutes at RT.

3. Wash the cells once with blocking buffer for 5 minutes at RT. Incubate the cells with primary antibody in $250 \mu \mathrm{L}$ blocking buffer for $16-24$ hours at $4^{\circ} \mathrm{C}$.

4. Wash the cells 3 times with blocking buffer for 5 minutes, each time, at RT. Incubate the cells with secondary antibody and nuclear stain in $250 \mu \mathrm{L}$ blocking buffer for 1 hour at RT in the dark.

5. Wash the cells 3 times with blocking buffer for 5 minutes at RT.

6. Place the coverslips face down in 1-2 drops of mounting medium on a microscope cover glass. Cover with a microscope slide and turn over. The cells should be directly beneath the cover glass.

\subsection{Fluorescence microscopy of IMCD cells}

Image cells that are positive for $\beta$ arr2-GFP and Sstr3-Myc and capture multiple focal planes (Z-stack) spaced at $\sim 0.3 \mu \mathrm{m}$ intervals. The GFP, Myc and DRAQ5 are excited with the 488, 552 and $638 \mathrm{~nm}$ laser lines, respectively.

Representative images of $\beta$ arr2-GFP localization in IMCD cells expressing Sstr3-Myc in cilia are shown in Fig. 5. After 20 minutes of vehicle treatment $\beta$ arr2-GFP remains localized throughout the cytoplasm, but is not detected within cilia (Fig. 5A). After 20 minutes of 10 $\mu \mathrm{M}$ SST treatment, $\beta$ arr2-GFP shows robust ciliary localization (Fig. 5B), suggesting that $\beta$ arr2-GFP localizes to cilia in response to Sstr3 activation.

\subsection{Transfection of hippocampal neurons with plasmid DNA}

1. To plate hippocampal neurons on glass-bottomed dishes, resuspend pelleted neurons from the hippocampus of one mouse in $1 \mathrm{~mL}$ of Neurobasal-A medium and slowly pipette the cells onto the glass surface until the medium resembles a blister and covers 
the entire glass surface. (see Note 12). Gently place the plate in a tissue culture incubator and allow the neurons to adhere for approximately 2 hours. Once the neurons have adhered, add $2 \mathrm{ml}$ of additional Neurobasal-A medium to completely cover the bottom of the dish.

2. On the day of transfection (we normally transfect on day 6), pipette virtually all the media from the dish into a sterile tube and keep it at $37^{\circ} \mathrm{C}$. (see Note 13 ).

3. Prepare the transfection mix. For each glass-bottomed dish, mix $1 \mu \mathrm{g}$ of DNA (500 ng of $\beta$ arr2GFP $+500 \mathrm{ng}$ of Sstr3DsRed) with $1 \mu \mathrm{l}$ of PLUS reagent in $200 \mu \mathrm{L}$ of OptiMEM and incubate for 5 minutes at RT. After 5 minutes, add $1.5 \mu \mathrm{L}$ of Lipofectamine LTX reagent, mix, and incubate for an additional 30 minutes at RT.

4. Pipette the transfection mix over the neurons and place the dish back in the incubator. After 2-4 hours, add back the original conditioned media recovered in step 3.13.2.

5. Incubate the cells with transfection mix for 16-24 hours before live-cell imaging analysis.

\subsection{Live-cell imaging of Sstr3-mediated recruitment of $\beta$ arr2-GFP in hippocampal neurons}

1. Prior to imaging the cells, replace the media with $2 \mathrm{~mL}$ of Hibernate A low fluorescence medium supplemented with B-27, glutaMAX and ITS. (see Note 14).

2. Image neurons expressing $\beta$ arr2-GFP and Sstr3-DsRed. Acquire Z-stacks of the red and green fluorescence sequentially at 30 second intervals for 10 minutes using a 0.2 $\mu \mathrm{m}$ step size and a 100x/1.4NA PlanApo VC oil immersion objective. The GFP and DsRed 2 are excited with the $488 \mathrm{~nm}$ and $561 \mathrm{~nm}$ laser lines, respectively.

3. Add somatostatin ( $20 \mu \mathrm{L}$ of $1 \mathrm{mM}$ stock solution) directly to the $2 \mathrm{~mL}$ of medium in the dish with a pipette and continue acquiring Z-stacks sequentially for 10 minutes.

An example of SST treatment of a hippocampal neuron expressing $\beta$ arr2-GFP and Sstr3DsRed is shown in Fig.6. Before SST treatment (t0) $\beta$ arr2-GFP is enriched at the base of the cilium but is not detected within the cilium. $\beta$ arr2-GFP localization in the cilium is detected within 2 minutes (t2) of SST treatment and is more robustly detected after 8 minutes (t8).

In conclusion, our experiments utilizing fixed and living cells indicate that exogenous $\beta$ arr 2 is enriched at the base of cilia expressing exogenous Sstr3 but is not detectable within the ciliary compartment. However, upon agonist addition, $\beta$ arr2 is rapidly recruited into cilia where it likely interacts with Sstr3 to modulate receptor signaling and/or trafficking. 


\section{Notes}

1. Permeabilization buffer should be stored at $-20^{\circ} \mathrm{C}$ in aliquots and thawed and kept at $4^{\circ} \mathrm{C}$ upon use.

2. The addition of sodium azide prevents contamination and allows the buffer to be stored at $4^{\circ} \mathrm{C}$.

3. This step is important to stick the coverslips to the bottom of the wells and to avoid their overlap.

4. Move gently your plate in perpendicular directions in order to disperse cells or the transfection mix in the wells. Avoid circular movements; otherwise, generated vortex will deposit cells or the transfection medium mostly in the middle of the well.

5. This overnight treatment with nocodazole will depolymerize microtubules and will therefore arrest mitotic cells in $\mathrm{G}_{2} / \mathrm{M}$ phase.

6. To avoid cell detachment, do not put directly the new medium onto the cells but slowly along wells walls. Never aspirate the liquid directly over the coverslips but lean the plate towards you and use a $200 \mu \mathrm{L}$ pipet tip to aspirate the medium along the wells walls.

7. In case of doubt about the coverslip orientation, cells covered surface can be observed on by holding it with the forceps and by exposing it to a source of light or by making a little scratch on the surface.

8. This step is required when using mounting media which do not dry (Glycerol-based). Avoid small holes that will favor oil contact with mounting media and the cells. Avoid coverslip movements on the slide, otherwise the cells will detach.

9. Do not forget to adapt the transfection mix to the volume of the used wells.

10. The centrosome can move in the horizontal and/or vertical axis during acquisition.

11. The antibody against acetylated- $\alpha$-tubulin allows visualization of stable MTs and, in addition to the axoneme of primary cilia, will also stain other stabilized MT such as cytoplasmic MTs (cell type dependent), mitotic spindle, and intercellular bridges. If cytoplasmic staining with this antibody is too strong and then avoid visualization of cilia, other ciliary markers can be used (Arl13b, glutamylated tubulin (GT335)).

12. Glass-bottom dishes are manufactured by drilling a hole in the bottom of a plastic 35 $\mathrm{mm}$ tissue culture dish and covering the hole by attaching a glass coverslip to the outside of the dish. Thus, a shallow well is formed in the middle of the dish which will retain the medium containing cells over the glass surface. 
13. Neurons are sensitive to media changes and this conditioned media will be added back to the neurons. Ensure that a small amount of media is retained in the glass-bottomed well to prevent the neurons from drying out. This reduction in volume at the beginning of the transfection appears to facilitate delivery of the DNA to the neurons and increases the transfection efficiency.

14. Hibernate medium allows the neurons to be maintained at $37^{\circ} \mathrm{C}$ in ambient $\mathrm{CO}_{2}$. 


\section{References}

1. Ishikawa, H. and Marshall, W. F. (2011) Ciliogenesis: building the cell's antenna. Nat. Rev. Mol. Cell Biol. 12, 222-234

2. Mitchison, H. M. and Valente, E. M. (2016) Motile and non-motile cilia in human pathology: from function to phenotypes. J. Pathol. 241, 294-309

3. Bornens, M. (2012) The centrosome in cells and organisms. Science 335, 422-426

4. Benmerah, A. (2013) The ciliary pocket. Curr. Opin. Cell Biol. 25, 78-84

5. Takao, D. and Verhey, K. J. (2016) Gated entry into the ciliary compartment. Cell. Mol. Life Sci. CMLS 73, 119-127

6. Lechtreck, K. F. (2015) IFT-Cargo Interactions and Protein Transport in Cilia. Trends Biochem. Sci. 40, 765-778

7. Schou, K. B., Pedersen, L. B., and Christensen, S. T. (2015) Ins and outs of GPCR signaling in primary cilia. EMBO Rep. 16, 1099-1113

8. Mykytyn, K. and Askwith, C. (2017) G-Protein-Coupled Receptor Signaling in Cilia. Cold Spring Harb. Perspect. Biol.

9. Dawson, T. M., Arriza, J. L., Jaworsky, D. E., Borisy, F. F., Attramadal, H., Lefkowitz, R. J., and Ronnett, G. V. (1993) Beta-adrenergic receptor kinase-2 and beta-arrestin-2 as mediators of odorant-induced desensitization. Science 259, 825-829

10. Molla-Herman, A., Boularan, C., Ghossoub, R., Scott, M. G. H., Burtey, A., Zarka, M., Saunier, S., Concordet, J.-P., Marullo, S., and Benmerah, A. (2008) Targeting of betaarrestin2 to the centrosome and primary cilium: role in cell proliferation control. PloS One 3, e3728

11. Kovacs, J. J., Whalen, E. J., Liu, R., Xiao, K., Kim, J., Chen, M., Wang, J., Chen, W., and Lefkowitz, R. J. (2008) Beta-arrestin-mediated localization of smoothened to the primary cilium. Science 320, 1777-1781

12. Mick, D. U., Rodrigues, R. B., Leib, R. D., Adams, C. M., Chien, A. S., Gygi, S. P., and Nachury, M. V. (2015) Proteomics of Primary Cilia by Proximity Labeling. Dev. Cell 35, 497-512

13. Pal, K., Hwang, S.-H., Somatilaka, B., Badgandi, H., Jackson, P. K., DeFea, K., and Mukhopadhyay, S. (2016) Smoothened determines $\beta$-arrestin-mediated removal of the $G$ protein-coupled receptor Gpr161 from the primary cilium. J. Cell Biol. 212, 861-875

14. Green, J. A., Schmid, C. L., Bley, E., Monsma, P. C., Brown, A., Bohn, L. M., and Mykytyn, K. (2016) Recruitment of $\beta$-Arrestin into Neuronal Cilia Modulates Somatostatin Receptor Subtype 3 Ciliary Localization. Mol. Cell. Biol. 36, 223-235 
15. Nager, A. R., Goldstein, J. S., Herranz-Pérez, V., Portran, D., Ye, F., Garcia-Verdugo, J. M., and Nachury, M. V. (2017) An Actin Network Dispatches Ciliary GPCRs into Extracellular Vesicles to Modulate Signaling. Cell 168, 252-263.e14

16. Kang, D. S., Tian, X., and Benovic, J. L. (2014) Role of $\beta$-arrestins and arrestin domaincontaining proteins in $\mathrm{G}$ protein-coupled receptor trafficking. Curr. Opin. Cell Biol. 27, $63-71$

17. Shankar, H., Michal, A., Kern, R. C., Kang, D. S., Gurevich, V. V., and Benovic, J. L. (2010) Non-visual arrestins are constitutively associated with the centrosome and regulate centrosome function. J. Biol. Chem. 285, 8316-8329

18. Piel, M., Meyer, P., Khodjakov, A., Rieder, C. L., and Bornens, M. (2000) The respective contributions of the mother and daughter centrioles to centrosome activity and behavior in vertebrate cells. J. Cell Biol. 149, 317-330

19. Taipale, J., Chen, J. K., Cooper, M. K., Wang, B., Mann, R. K., Milenkovic, L., Scott, M. P., and Beachy, P. A. (2000) Effects of oncogenic mutations in Smoothened and Patched can be reversed by cyclopamine. Nature 406, 1005-1009 
Figure Legends

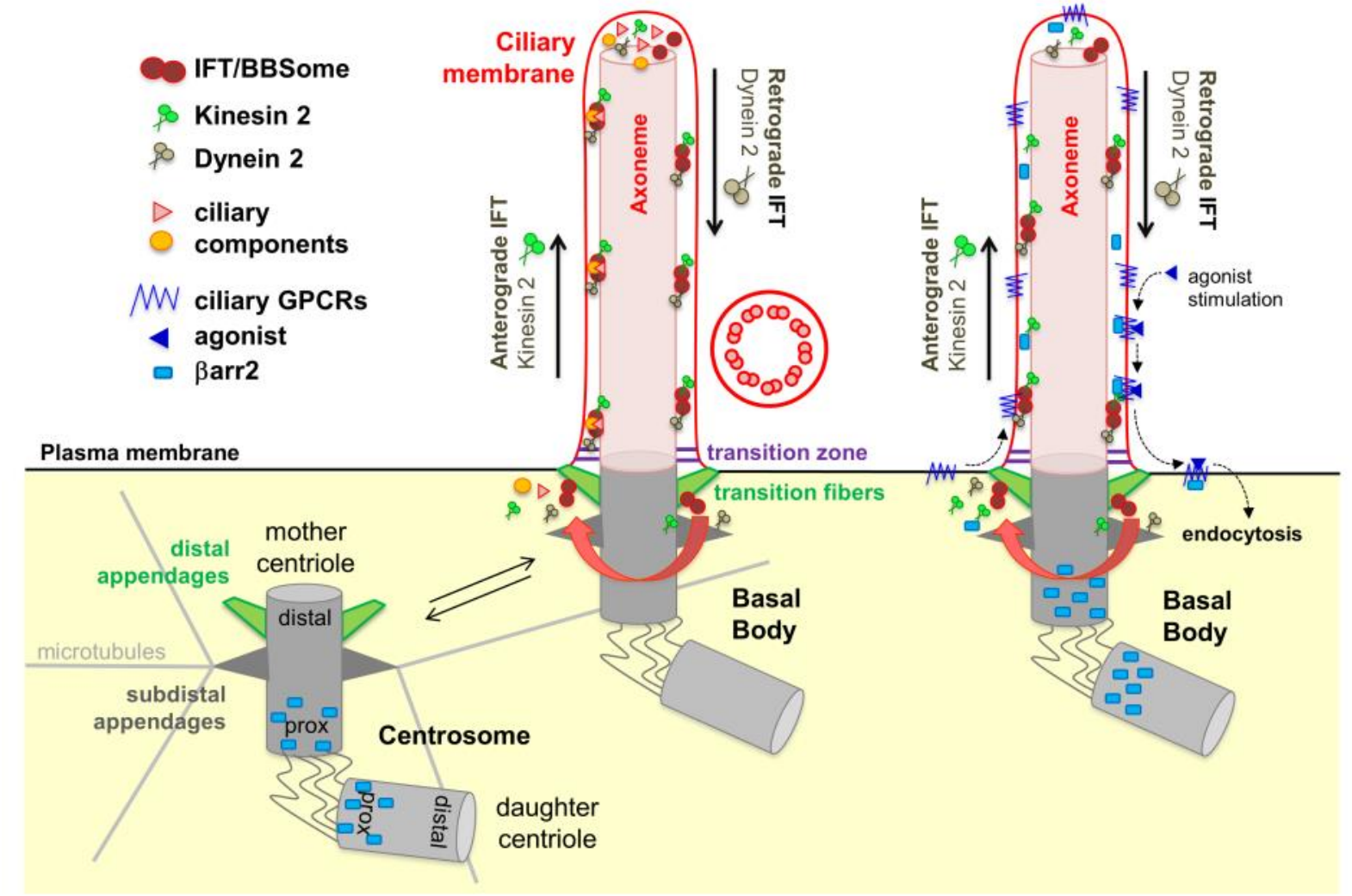

Figure 1. Organization of centrosome and primary cilium.

The centrosome in interphasic cells is composed of two centrioles (mother and daughter). The mother is characterized by the presence of the subdistal appendages on which microtubules are anchored and of distal appendages which are required for docking onto cellular membranes during ciliogenesis. Ciliogenesis of primary cilia occurs in quiescent or terminally differentiated $\mathrm{G}_{0}$ cells in which the centrosome docks onto membranes and becomes the basal body. The axoneme is composed of nine doublets of microtubules extended from the mother centriole. The ciliary membrane presents a specific composition thanks to the presence of the transition zone and of transition fibers which act as filter/diffusion barriers and prevent free exchange between the cilium compartment and the rest of the cell. Ciliogenesis and transport in and out of the cilium requires specific machinery, called IFT, which mediates anterograde and retrograde transport. The ciliary membrane is highly enriched in GPCRs. GPCRs are imported into and exported out of cilia through mechanisms involving IFT, the BBSome and Barr2. Stimulation of ciliary GPCRs by their agonist induces $\beta$ arr2 recruitment to activated GPCRs and potentially facilitates the association with retrograde IFT/BBSome for ciliary export. 


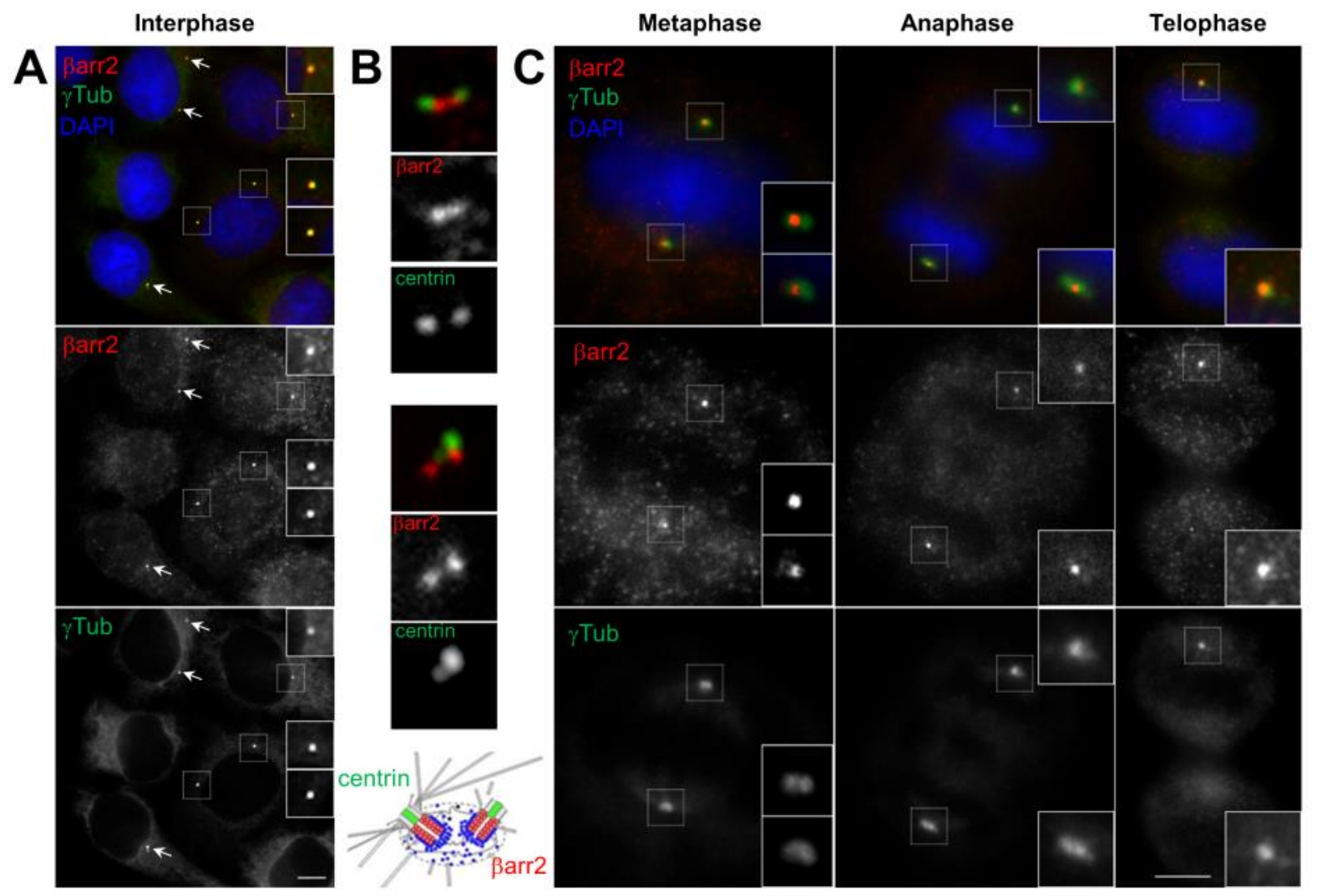

Figure 2. Endogenous $\beta$ arr2 is localized at the centrosome during the overall cell cycle.

(A) Synchronized HeLa cells were fixed and stained for endogenous $\beta$ arr2 (rabbit pan-arrestin (Abcam)) and for $\gamma$-tubulin ( $\gamma$ Tub). DNA was stained with DAPI. Representative images of interphase and mitosis stages are shown. Arrows indicate representative $\gamma$ Tub-stained centrosomes. Insets show higher magnifications of representative areas. (B) HeLa cells stably expressing a centrin-GFP were fixed and stained for endogenous $\beta$ arr2 (goat anti-beta arrestin 2 (Abcam)) or transfected with plasmids encoding for the Barr2-Cherry fusion, then fixed and directly observed. The possible distribution of $\beta$ arr2 within the centrioles is illustrated:

Centrin is localized at the distal regions of the centrioles (green). $\beta$ arr2 is likely localized at the proximal region of the centrioles (red). Pericentrin is localized at the pericentriolar matrix around centrioles (blue). (C) Hela cells were synchronized, fixed and stained for $\beta$ arr2 and $\gamma$-tub. Different mitotic stages are shown. Insets show higher magnifications of representative centrosomal regions. Scale bars represent $5 \mu \mathrm{m}$. 


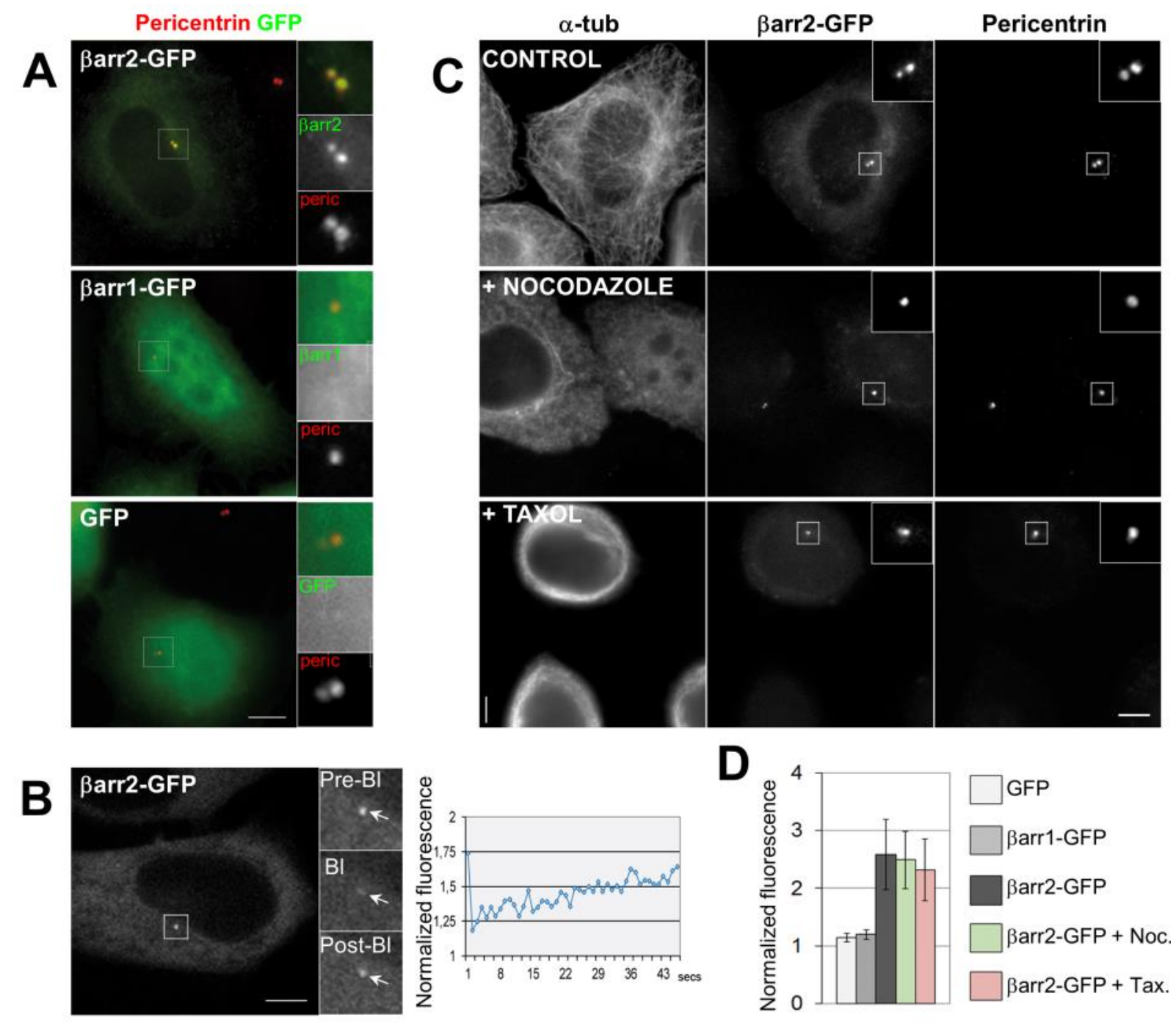

Figure 3. Barr2 is targeted and enriched at the centrosome independently of microtubules. (A) HeLa cells were transfected with plasmids encoding for Barr2-GFP, Barrl-GFP or with GFP alone, fixed and stained for the centrosomal marker pericentrin. Insets show higher magnifications of centrosomal regions. (B) Live HeLa cells transiently expressing $\beta a r r 2-G F P$ were treated for $1 \mathrm{~h}$ at $37^{\circ} \mathrm{C}$ with Nocodazole $(10 \mu \mathrm{M})$ then used for FRAP experiments. Images of a representative cell are shown. The centrosomal region was bleached twice. Insets show higher magnifications of the bleached region before bleaching (Pre-Bl), just after bleaching (Bl) and after bleaching (Post-Bl). Arrows indicate the analyzed Barr2-GFP stained centrosome. Fluorescence intensity of $\beta a r r 2-G F P$ at the centrosome was normalized to cytoplasmic staining within an identical cytoplasmic region. (C) HeLa cells expressing Barr2-GFP were untreated (control) or treated with Nocodazole or Taxol $(10 \mu \mathrm{M})$ to depolymerize or stabilize microtubule network, respectively. Cells were fixed and stained for microtubules ( $\alpha$-tubulin) and for the centrosome (Pericentrin). Insets show higher magnifications of centrosomal regions. (D) Centrosome-associated GFP fluorescence was quantified using a region defined by pericentrin staining and normalized to the cytoplasmic signal within the same cells. Values are the means (+/-2 SD) of at least 20 cells from three independent experiments. Scale bars represent $5 \mu \mathrm{m}$. 

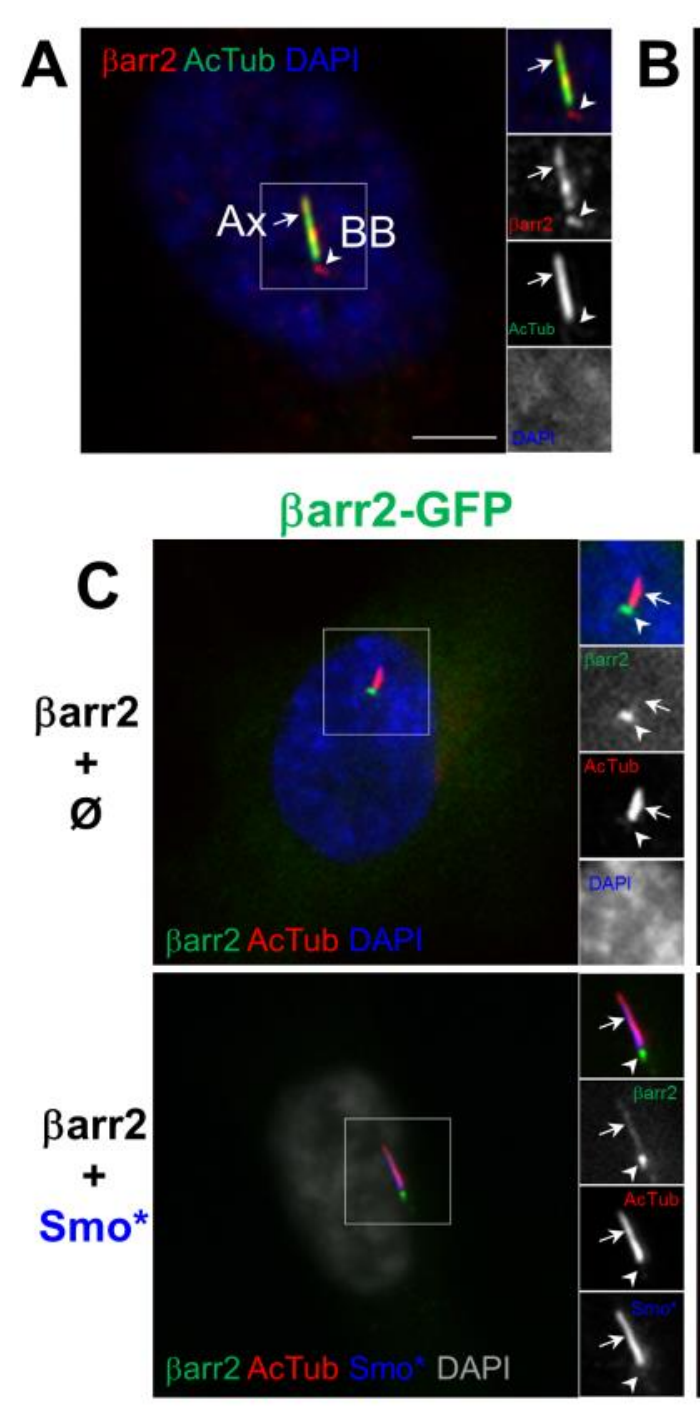
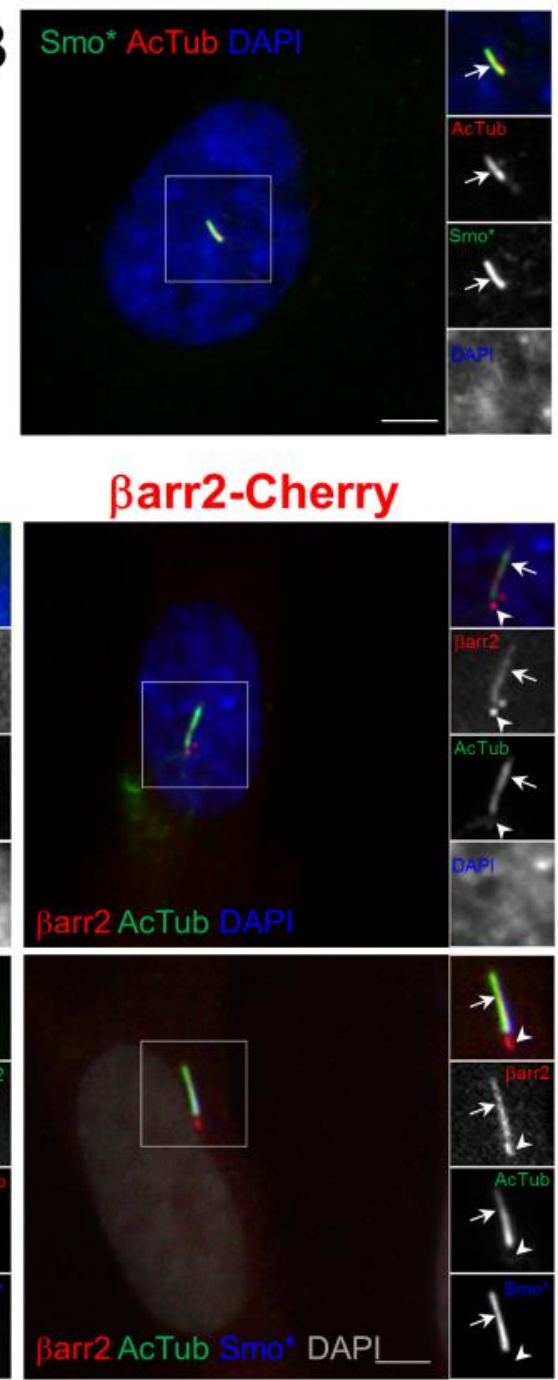

\section{Figure 4. $\beta$ arr2 is found in the axoneme and basal body of primary cilia.}

(A) Confluent RPE1 cells were serum-starved for 24 hours, then fixed and stained for acetylated- $\alpha$-tubulin (AcTub, red), which is highly enriched in primary cilia (axoneme (Ax)) and for endogenous $\beta$ arr2 (rabbit pan-arrestin (Abcam)). Arrows and arrowheads indicate AcTub-stained axonemes and $\beta$ arr2 stained basal bodies (BB), respectively. (B) RPE1 cells transfected with a plasmid encoding for a Flag-tagged active form of Smoothened (Smo*), were serum-starved for 24 hours after transfection, then fixed and stained for AcTub and Flag (Smo*). (C) RPE1 cells transfected with plasmids encoding either $\beta$ arr2-GFP (left panels, green) or $\beta$ arr2-Cherry (right panels, red) fusions alone (upper panels) or together with Flagtagged Smo* (lower panels) were serum-starved for $24 \mathrm{~h}$ after transfection, then fixed and stained for either AcTub (upper panels) or for AcTub and Flag (Smo*, lower panels). Insets show higher magnifications of representative areas. Scale bars represent $5 \mu \mathrm{m}$. 

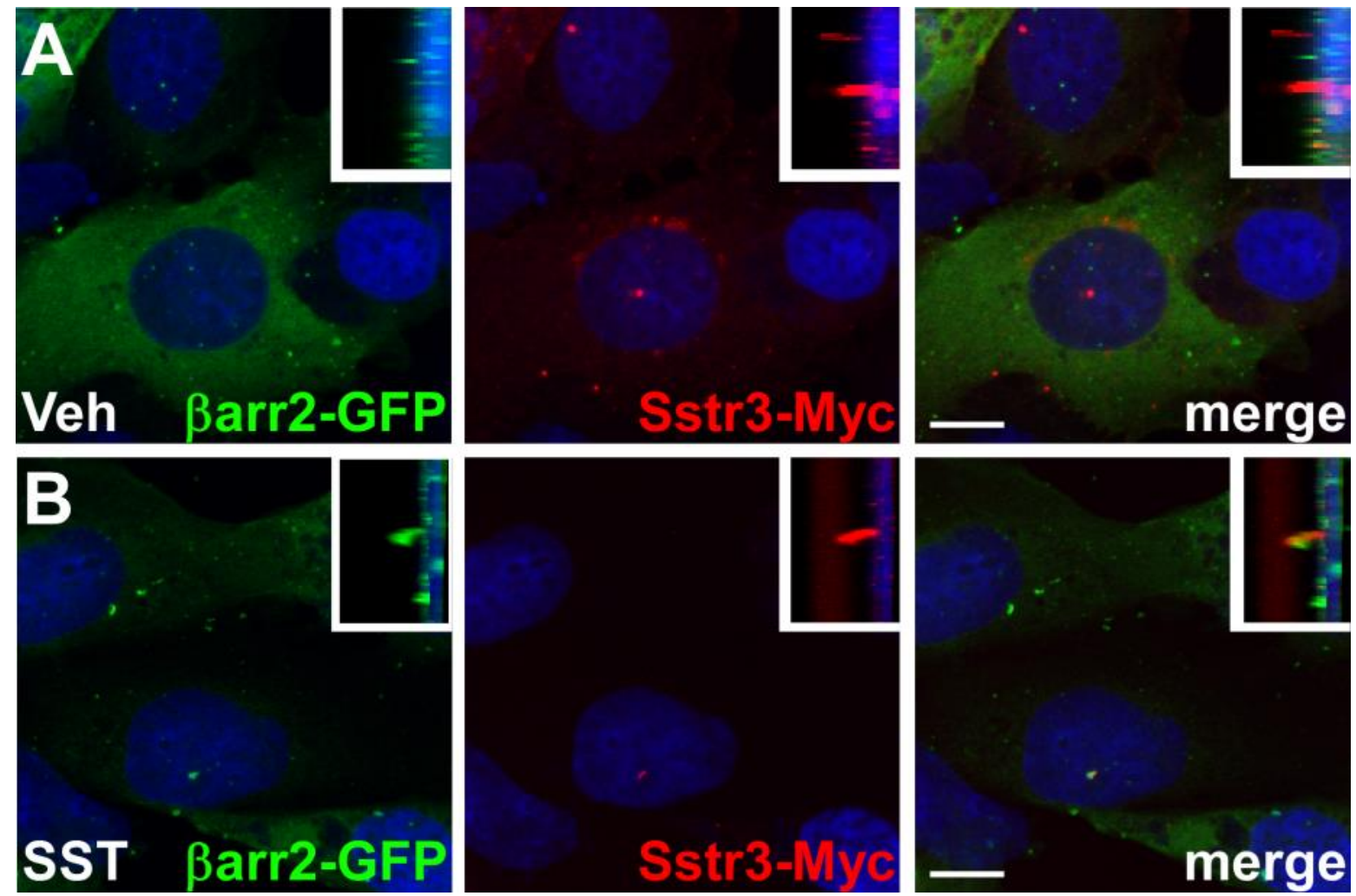

Figure 5. $\beta$ arr2-GFP is recruited into cilia in IMCD cells in response to somatostatin treatment.

IMCD cells expressing $\beta$ arr2-GFP and Sstr3-Myc were treated with vehicle (Veh) (A) or 10 $\mu \mathrm{M}$ somatostatin (SST) (B) for 20 minutes, fixed and labeled with an antibody to detect Myc. Insets show side views of the cilia. Nuclei were stained with DRAQ5. Scale bars represent 10 $\mu \mathrm{m}$. 


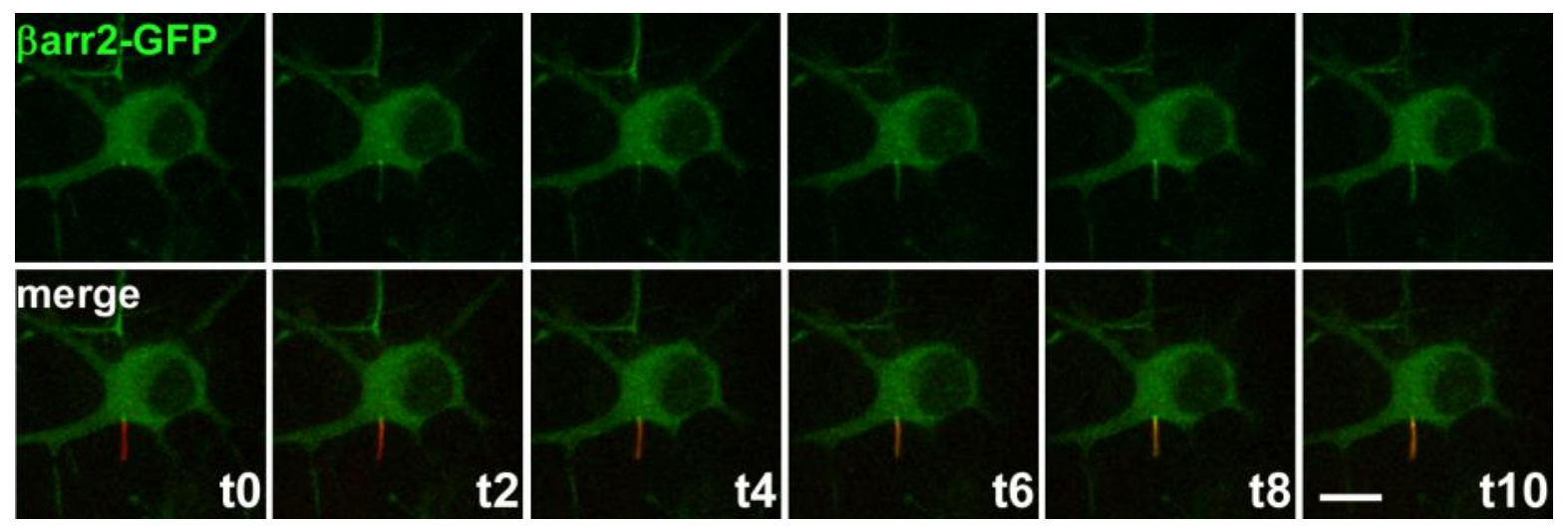

Figure 6. Barr2-GFP is recruited into cilia in hippocampal neurons in response to somatostatin treatment.

Live hippocampal neurons expressing $\beta$ arr2-GFP and Sstr3-DsRed2 were treated with $10 \mu \mathrm{M}$ somatostatin (SST) and imaged for 10 minutes. Elapsed time (t) in minutes of SST treatment is indicated in the lower right corner of each panel. Each image is a maximum intensity projection of a z-stack that encompassed the entire cell body and cilium. Upper panels show the green channel ( $\beta$ arr2-GFP) and lower panels show both the green and red (Sstr3-DsRed) channels. Scale bar represents $10 \mu \mathrm{m}$. 